Journal of the Magnetics Society of Japan Vol. 15 Supplement, No. S2 (1991)

(C) 1991 by The Magnetics Society of Japan

\title{
ANALYSIS OF UNUSUAL TRANSITION OF CO-Cr MEDIUM IN SINUSOIDAL WAVE RECORDING USING RING HEAD
}

\author{
Takanori SATO, Hiroshi UCHIYAMA and Naoki HONDA \\ R\&D division, Sony Magnetic Products Inc. \\ 3-4-1 Sakuragi, Tagajo, Miyagi, 985, JAPAN
}

\begin{abstract}
The Co-Cr perpendicular medium shows superior recording characteristics using a ring head. We observed an unusual reproduced waveform for a transition in sinusoidal recording which is quite different from a usual dipulse. The phenomenon became apparent with lower recording current levels and longer wavelengths. The waveform of the unusual transition was analysed using a waveform transition based on the Hilbert transformation. We determined that the transition consisted of one main pulse followed by two sub-pulses. We propose a simple model for the unusual transition.
\end{abstract}

\section{INTRODUCTION}

It is known that the $\mathrm{Co}-\mathrm{Cr}$ perpendicular magnetic recording medium shows high density recording characteristics even when a ring head is used [1]. In the analog FM recording with VTRs, a sinusoidal wave is widely used as the recording signal.

The reproduced waveforms corresponding to the magnetization transitions in the recording of sinusoidal signals on a $\mathrm{Co}-\mathrm{Cr}$ medium using a ring head are usually dipulse. We have found, however, an unusual complicated reproduced signal in sinusoidal wave recording at low frequencies and low recording current levels. We suppose that the phenomenon is related to the behavior of perpendicular magnetic recording using a ring head.

In this paper, the unusual transition is analysed and a simple model for the transition is proposed.

\section{EXPERIMENTAL}

The Co-Cr perpendicular magnetic recording medium was prepared by DC magnetron sputtering using a polyimide base film. The $\mathrm{Co}-\mathrm{Cr}$ medium is overcoated with a $0.01 \mu \mathrm{m}$-thick protective layer of sputtered carbon and formed as a stretched disk [3] .

Table I shows the medium and head specifications. The ring head used was a metal-in-gap type made of high Bs metal [2] . A head with a gap length of $0.15 \mu \mathrm{m}$ was used in waveform analysis and a head with a gap length of $0.29 \mu \mathrm{m}$ was used to observe the effect of recording current and wavelength.

The head for the stretched disk is composed of a small head tip suspended by a

Table I: Properties of medium and head

\begin{tabular}{lcc}
\hline Co-Cr medium & MIG head \\
Thickness: $0.2 \mu \mathrm{m}$ & Bs of metal: & $14.5 \mathrm{kG}$ \\
Hc(perp.): 1250 Oe & Gap length : & $0.29,0.15 \mu \mathrm{m}$ \\
Ms : 400 emu/cc & Track width: & $30 \mu \mathrm{m}$ \\
\hline
\end{tabular}


pair of parallel thin-plate springs [3] .

The relative head-to-medium velocity was $3 \mathrm{~m} / \mathrm{s}$ in the recording measurement.

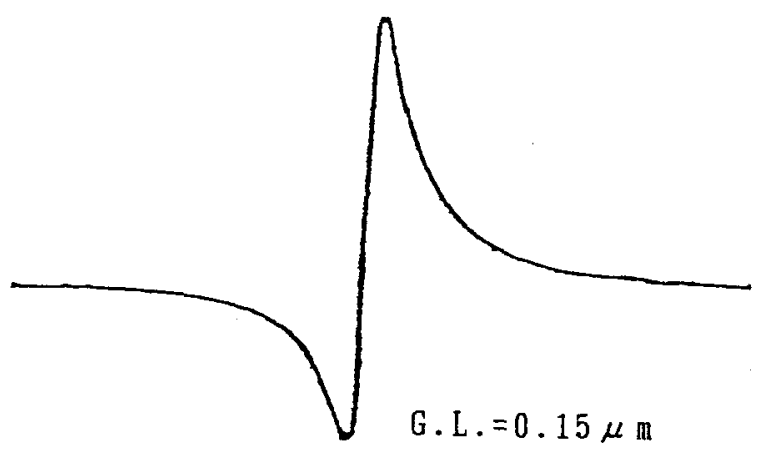

(a) Usual transition

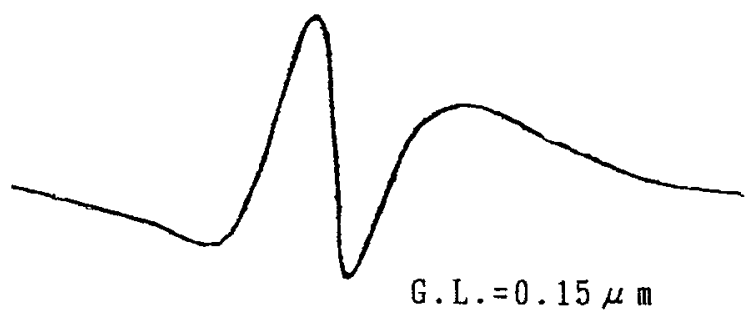

(b) Unusual transition

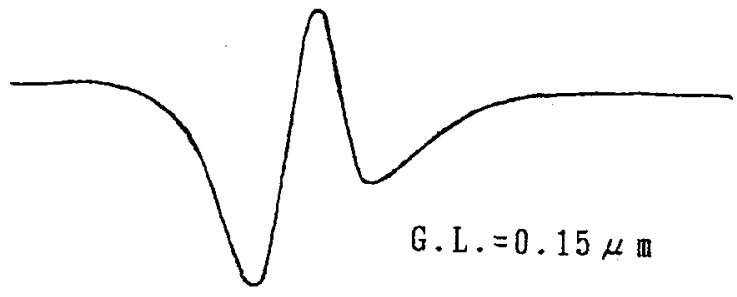

(c) Hilbert transformed waveform of unusual transition

Fig. 1 Waveforms of usual and unusual transition.
The reproduced waveform was digitized in 1000 points in a time domain of $2 \mathrm{nsec}$ by a digital oscilloscope and the waveform was analysed using a personal computer.

Figure 1 (a) and (b) show the usual dipulse waveform and the unusual reproduced waveform in sinusoidal wave recording, respectively. The unusual reproduced waveform was observed at $0.3 \mathrm{AT}_{\square-\mathrm{P}}$ recording current and the dipulse waveform appeared at $0.8 \mathrm{AT} \mathrm{T}_{-\mathrm{F}}$ recording current at the signal frequency of $0.3 \mathrm{MHz}$. The unusual waveform was clearly observed at low current levels in addition to at low frequencies, so the effect of the recording current level and the frequency on the occurrence of the unusual waveform could be investigated in detail.

A typical reproduced unusual waveform was analysed using a waveform transformation based on a Hilbert transformation using an FFT.

\section{RESULT AND DISCUSSION}

\section{Effect of recording current and wavelength}

In the recording on a $\mathrm{Co}-\mathrm{Cr}$ medium using a ring head, the reproduced waveform of long wavelength even in sinusoidal wave recording is usually dipulse. But unusual reproduced wave forms are observed when the recording current is low.

First, we observed the effect of the recording wavelength on the waveform while the recording current level was kept constant. It is known that an unusual wave form was first noticeably seen at a long wavelength and then became clear with the wavelength.

Next, the recording current and wavelength under which the unusual transition occurred were investigated regarding to the recording current and wavelength. The result is summarized in Fig.2. The unusual transition was observed at longer wavelengths and lower currents.

A typical change of waveforms with recording current is shown Photo 1.

The threshold of the occurrence is expressed 
by $\mathrm{I} / \lambda \div 0.04(\mathrm{AT}-s / \mu \mathrm{m})$, where $\mathrm{I}$ and $\lambda$ denote the recording current and the wavelength, respectively. Then we can extrude the undesirable phenomenon by choosing a recording current and wavelength greater than this value.

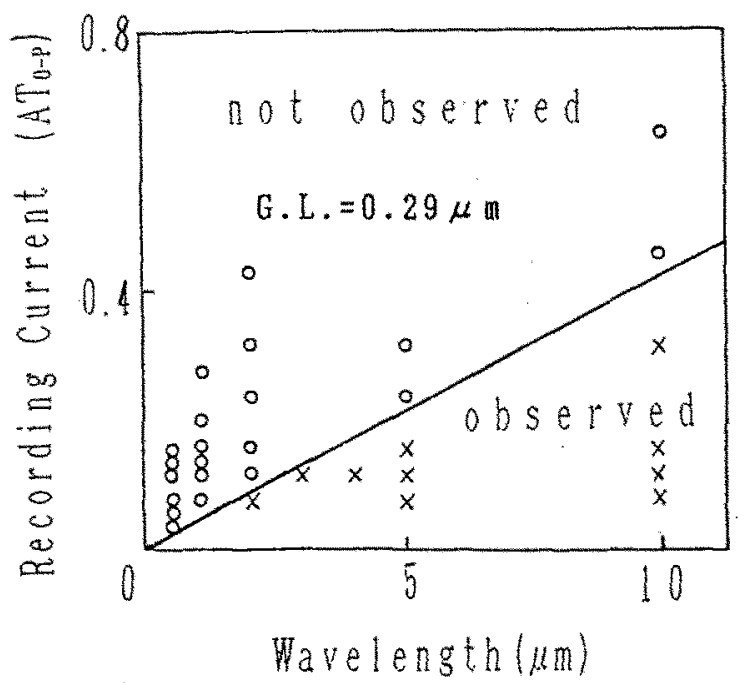

Fig.2 Mapping of unusual transition for wavelength and current.

\section{Structure of unusual transition}

As shown in Fig.1(b), the unusual reproduced waveform is composed of some dipulses of some transitions of perpendicular magnetization. The unusual waveform was analysed using a waveform transformation. A dipulse waveform of a perpendicular recording is transformed to a single pulse by a Hilbert transformation. We determined the appropriate angle for the Hilbert transformation to be 80 degrees in the present case by a phase characteristics analysis of an isolated pulse of a rectangular wave recording $[4]$. The transformed waveform is shown in Fig.1(c). This figure shows that the transformed waveform consists of three symmetric single pulses.

Next, we tried to separate three single pulses in the transformed waveform. Figure 3 shows the result of the separation. Here each

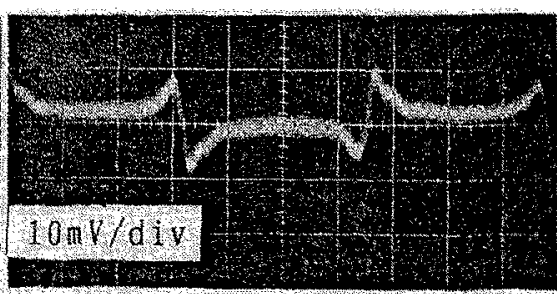

(a) $\lambda=10 \mu \mathrm{m} \quad \mathrm{I}=0.8 \mathrm{AT}$

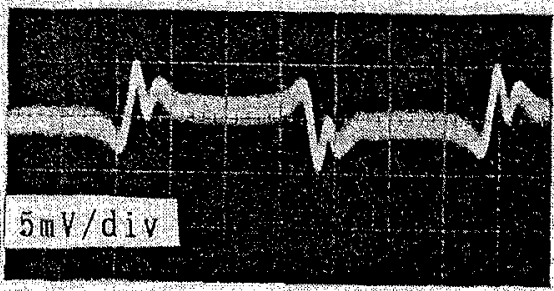

(b) $\lambda=10 \% \mathrm{~m}, \mathrm{~T}=0,4 \mathrm{AT}$

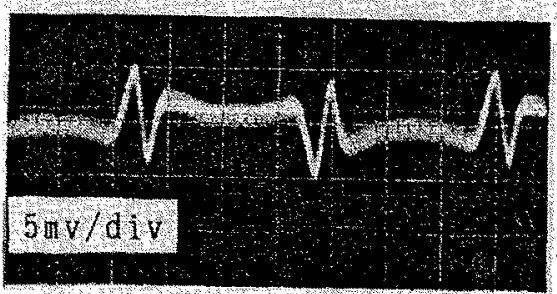

(c) $\lambda=10, \mu \mathrm{m} \quad \mathrm{I}=0.2 \mathrm{AT}$

Photo 1 Waveforms in sinusoidal wave recording with decreased current. (G. L. $=0.29 \mu$ i $)$

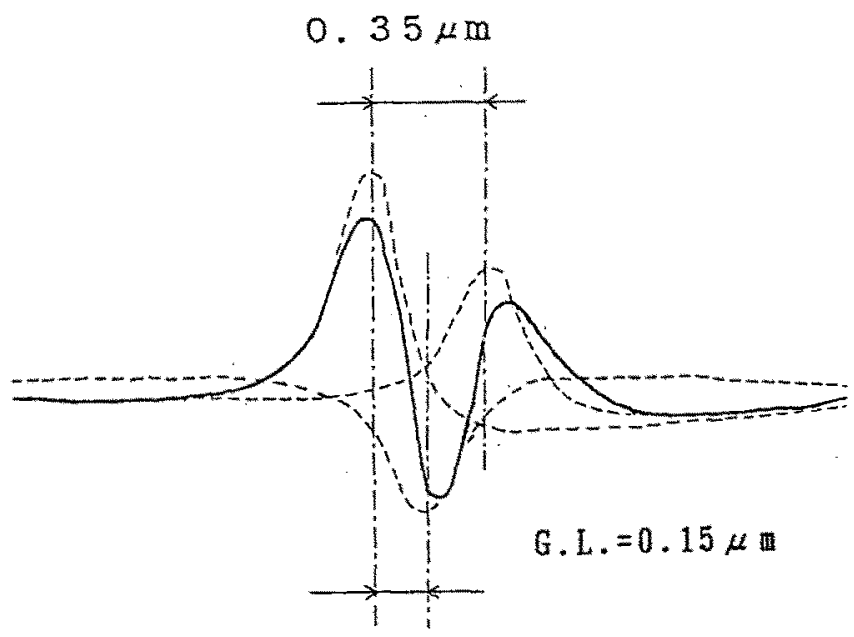

$0.175 \mu \mathrm{m}$

Fig.3 Transformed waveform and separated three single pulses. 
separated waveform is of the Hilbert transformed from the dipulse wave form of rectangular wave recording, and only the amplitude and the position of the form are changed in the fittins process. From the sepatation, we determined that unusual waveform consists of one main pulse followed by two sub-pulses. The height ratio of the main pulse and two sub pulses are evaluated to be rhout $2: 1 ;$. The distance of the subpulses from the main pulse are estimated to be $0.175 \mu \mathrm{m}$ and $0.35 \mu \mathrm{m}$, respectively. We dedured a transition model corresponding to the mustal waveform as in Fig.4, where the magnelizalion arection is supposed to be almost perpendicular.

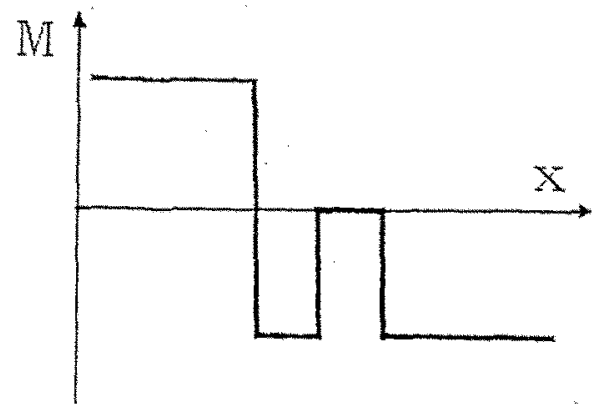

Fig.4 Magnetization reversal model for unusual transition

\section{Waveform in lower current}

The change of the reproduced waveform in lower recording currents than optimum was investigated.

The dependence of the dipulse ratio and the peak-to-peak pulse height of the wave form on the recording current is shown in Fig.5. The pulse height and dipulse ratio decreases with the recording current. While the peah height decreases to about a half of the optimum, the dipulke retio changes from about 0.7 to 0,5 . The change in the dipulse ratio, how ter, corresponds to the difference of only 15 degrees in the Hilbert transformation angle which transforms the dipulse to a symmetrical one. The deviation of

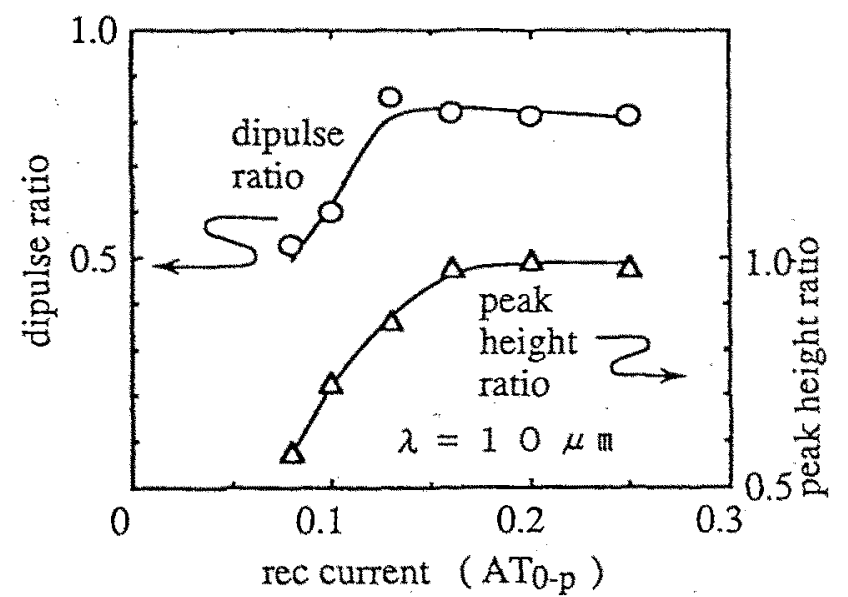

Eig.5 The current dependence of dipulse ratio and peak height ratio.

the angle has little effect on the transformed waveform.

Then, the above assumpution that the subpulse waveform can be fitted by choosing the amplitude of the isolated pulse waveform at the optimum recording current may be reasonable.

\section{Revording process}

The transition model shown in Fig. 4 can be understood based on the bi-direction recording character of a ring head.

The reconding process is shown in Fig. 6 . The recording ourrent changes slowly near zero cross level as in Fig.6 (a). Within the threshold level, it is assumed that the medium is not magnetized nor its recorded magnetization changed by the head field.

Step 1: The medium is magnetized in the negative direction on the leading edge of the head and in the positive direction on the trailing edge. The region masnetized negatively on the leading edge is magnetized positively after passing through on the trailing edge.

Step 2: The negative magnetization remains unchanged after passing through the trailing edge and no new magnetization occurs on the leading edge, because the magnetic head field strength is below the threshold on both edges. 


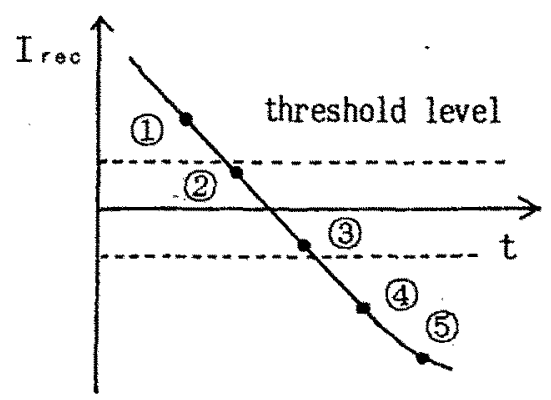

(a) current level

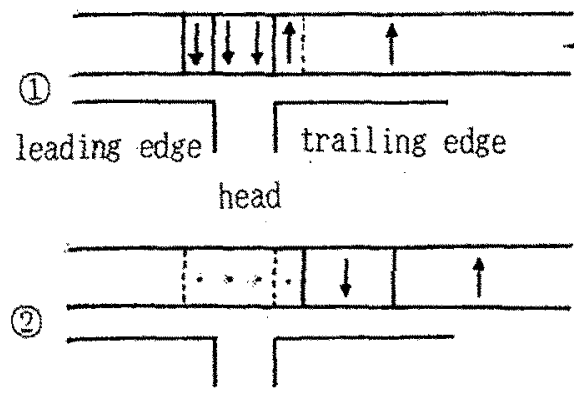

(3)

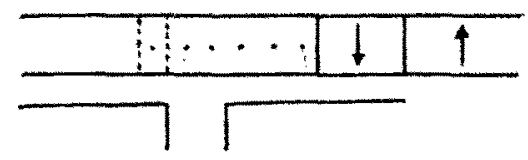

(4)

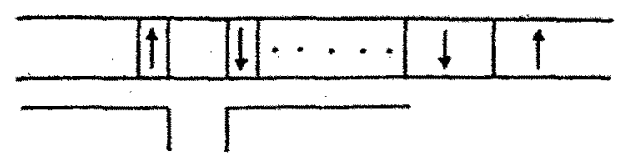

non-magnetized region

(5)
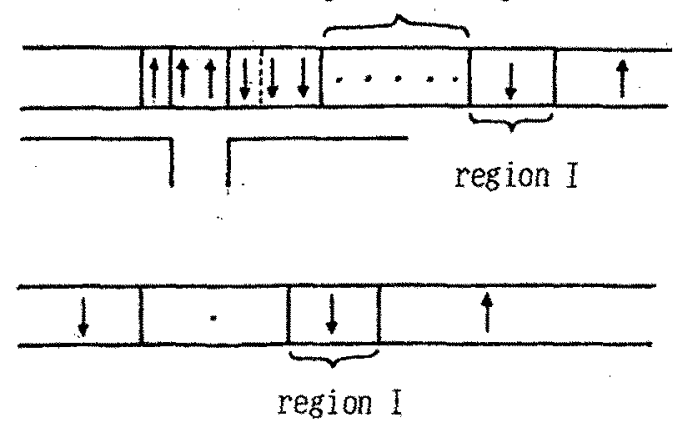

(b)

Fig.6 Recording process.
Step 3: The process continues in the same way as step 2. The non-magnetized region spread and passes through the trailing edge without being magnetized.

Step 4: The medium is magnetized again. But the direction of the magnetic head field is opposite to the direction in step 1.

Step 5: The rewriting on the trailing edge after writing on the leading edge continues.

Finally, the transition of magnetization as the last stage in Fig.6 is formed, which agrees with the model in Fig.4. It could be found that there is a non-magnetized region, which is imposed in the process between step 2 and step 3 . The non-magnetized region is expected to extend and affects the transition waveform as the rate of the current change becomes low, i.e. in longer wavelengths or lower current levels.

This simple model predicts that the width of region I corresponds to the length from the starting point of writing on the leading edge side to the starting point of writing on the trailing edge. The length is estimated to be about $0.18 \mu \mathrm{m}$, as mentioned previously. This means that the recording region of the head is restricted to about $0.03 \mu \mathrm{m}$ from gap edge in the present case.

The height ratio of the three pulses agrees at least qualitatively with that of the experiment. The deviation of the recorded magnetization direction and the unsaturated recording condition in the transition must be considered in a more the detailed analysis.

\section{SUMMARY}

An usual transition of $\mathrm{Co}-\mathrm{Cr}$ medium in a sinusoidal wave recording using a ring head was investigated. The unusual transition became clear for lower recording currents and longer wavelengths. The phenomenon could be avoided by the proper choice of the recording currext and wavelength. The transilion was determined to consist of one main transition followed by two sub-transitions by an analysis of the transition using a waveform transformation based on the Hilbert transformation. A simple 
model for the transition is proposed.

\section{ACKNOWLEDGEMENTS}

The authors wish to thank S. Ogata and T. Saito for preparing the head tips. They also thank $k$. Endo for his assistance with the recording measurements.

\section{REFERENCES}

[1] T. Suzuki and S. Iwasaki, Tech Meeting of IEICE, "RECORDING TO PERPENDICULAR MAGNETIC RECORDING MEDIA USING A RING TYPE HEAD" MR81-8 (1981) (in Japanese)

[2] S. Ogata, H. Uchiyama, N. Honda, and M. Hayakawa, Ann. Conf. IEICE Japan, "MIG HEAD USING HIGH BS METAL FOR PERPENDICULAR MAGNETIC RECORDING" 5-289, (1990) (in Japanese)

[3] N. Honda and H. Uchiyama, J of Mag. Soc. Japan, "A NEW STRETCHED-DISK INTERFACE FOR PERPENDICULAR MAGNETIC RECORDING USING CO-Cr MEDIUM" Vol.13 Sup.S1, pp.53 (1989)

[4] T. Sato, N. Honda, and H. Uchiyama, Ann. Conf. of IEICE Japan, "PEAK SHIFT CHARACTERISTICS OF CO-Cr PERPENDICULAR RECORDING MEDIUM USING A NARROW GAP RING HEAD" 5-34, (1991) (in Japanese) 